\section{Probabilistic Structured Query Methods}

\begin{abstract}
Structured methods for query term replacement rely on separate estimates of term frequency and document frequency to compute the weight for each query term. This paper reviews prior work on structured query techniques and introduces three new variants that leverage estimates of replacement probabilities. Statistically significant improvements in retrieval effectiveness are demonstrated for cross-language retrieval and for retrieval based on optical character recognition when replacement probabilities are used to estimate both term frequency and document frequency.
\end{abstract}

\section{KEYWORDS}

Structured queries, Cross-language information retrieval, Document image retrieval

\section{INTRODUCTION}

There are many situations in which it is desirable to match a query term with different terms in a document. Well known examples include stemming (where any word that shares the same stem should be matched), thesaurus expansion (where terms with similar meanings should be matched), and crosslanguage retrieval (where terms with similar meanings in different languages should be matched). When the mappings among matching terms are known in advance, the usual approach is to conflate the alternatives during indexing. That is the typical way in which stemming is implemented, for example. Query-time implementations are necessary when appropriate matching decisions depend on the nature of the query, as might be the case with systems that provide the searcher with interactive control over thesaurus expansion. In this paper, presently known techniques for query-time replacement are reviewed, new techniques that leverage estimates of replacement probability are introduced, and experiment results that demonstrate improved retrieval effectiveness in two applications (Cross-Language Information Retrieval (CLIR) and retrieval of scanned documents based on Optical Character Recognition (OCR)) are presented.

CLIR has received more attention than any other querytime replacement problem in recent years, and several effective techniques are now known. Query translation research has developed along two broad directions, typically referred to as "dictionary-based" and "corpus-based" techniques. Broadly speaking, corpus-based techniques seek to optimize retrieval effectiveness through reliance on observed translation probabilities in aligned corpora, while dictionary-based techniques are optimized for the case where reliable estimates of translation probability are not available.

A key idea in the so-called vector-space approach to information retrieval is reliance on two statistics: (1) term frequency $(T F)$, the number of occurrences of a term in a document, and (2) document frequency $(D F)$, the number of documents in which a term appears. $T F$ is a measure of aboutness, which has beneficial effects on both precision and recall. $D F$ is a measure of specificity, and its principal effect is on precision. In general, high $T F$ and low $D F$ are preferred, with the optimal combination of those factors typically being determined through experimentation (c.f., [14]).

Pirkola appears to have been the first to try separately estimating $T F$ and $D F$ for query terms in a CLIR application [13], using the InQuery synonym operator to implement what he called "structured queries." InQuery's synonym operator was originally designed to support monolingual thesaurus expansion, so it estimates $T F$ and $D F$ as follows [11]:

$$
\begin{aligned}
& T F_{j}\left(Q_{i}\right)=\sum_{\left\{k \mid D_{k} \in T\left(Q_{i}\right)\right\}} T F_{j}\left(D_{k}\right) \\
& D F\left(Q_{i}\right)=\left|\bigcup_{\left\{k \mid D_{k} \in T\left(Q_{i}\right)\right\}}\left\{d \mid D_{k} \in d\right\}\right|
\end{aligned}
$$

where $Q_{i}$ is a query term, $D_{k}$ is a document term, $T F_{j}\left(Q_{i}\right)$ is the term frequency of $Q_{i}$ in document $j, D F\left(Q_{i}\right)$ is the number of documents that contain $Q_{i}, d$ is a document, and $T_{j}\left(Q_{i}\right)$ is the set of known replacements (in this case, translations) for the term $D_{k}$. Essentially, these equations treat any occurrence of a replacement as an occurrence of the query term. This represents a very cautious strategy in which a high $D F$ for any replacement will result in a high $D F$ (and thus a low weight) for new joint $D F$ of that query term. Retrieval results are then dominated by query terms that have no "unsafe" (very common) replacements. For example, the Arabic query term " "علي" can either mean "on" or the proper name "Ali." If "Ali" appears in few documents but "on" appears in many, equation (2) will treat "علي" as if it were at least as common as "on." When there is not a large disparity in $D F$, equation (1) implements a kind of query expansion effect. For example, the Arabic word "خبز" can be translated as "bread" or "bake," and equation (1) would (with proper stemming) reward an occurrence of "baking bread."

Corpus-based approaches to CLIR have generally developed within a framework based on language modeling rather than vector space models, at least in part because modern statistical translation frameworks offer a natural way of integrating translation and language models [18]. In general, language modeling approaches to retrieval rely on collection frequency $(C F)$ in place of $D F:{ }^{1}$

$$
C F\left(Q_{i}\right)=\sum_{k \in C} T F_{k}\left(Q_{i}\right)
$$

where $\mathrm{C}$ represents the collection, and the other terms are as defined above. Whether $D F$ is better than $C F$ depends on how we model the searcher's task-when the goal is to find entire documents, $D F$ models the concept of "selectivity" with higher fidelity.

The next section introduces a set of replacement strategies that leverage observed replacement probabilities (from corpora) while retaining the vector space model's concept of $D F$. The effectiveness and efficiency (relative to present baselines) of this strategy is then shown in subsequent sections for two applications: CLIR, and retrieval from scanned documents using OCR. The paper then concludes with some notes on the limitations of the techniques presented here and opportunities for future work on this problem.

\footnotetext{
${ }^{1}$ Hiemstra's work is a notable exception [6].
} 


\section{BEYOND PIRKOLA'S METHOD}

Kwok was the first to introduce a variant to Pirkola's method, aiming to reduce implementation complexity by replacing the union operator with a sum [8]:

$$
D F\left(Q_{i}\right)=\sum_{\left\{k \mid D_{k} \in T\left(Q_{i}\right)\right\}} D F\left(D_{k}\right)
$$

An alternative approach, not previously explored, would be to use the maximum document frequency of any replacement (MDF):

$$
D F\left(Q_{i}\right)=\underset{\left\{k \mid D_{k} \in T\left(Q_{i}\right)\right\}}{M A X}\left[D F\left(D_{k}\right)\right]
$$

All three variants (Pirkola, Kwok, and MDF) lower bound the $D F$ for a query term by the $D F$ of its most common replacement, and the experiments reported in Sections 3 and 4 below show no statistically significant difference between the three techniques.

All three techniques treat every known replacement as equally likely. This risks a somewhat counterintuitive result: introduction of a translation dictionary with improved coverage of rare translations could actually harm retrieval effectiveness. To see this problem, consider a case a query term in which $99.9 \%$ of its instances should be translated as some rare term (e.g., "superfluous"), but in $0.1 \%$ of the cases a translation that happens to be a common term (e.g., "the") would actually be appropriate. In such cases, the common term leads to a high joint $D F$, effectively diminishing the value of the original query term. This exact situation actually arises often with dictionaries built from aligned corpora using statistical methods, since there is always some chance that any term might observed to be used as a replacement for any other term. One way to resolve the problem is to use a weighted variant of Kwok's method:

$$
D F\left(Q_{i}\right)=\sum_{\left\{k \mid D_{k} \in T\left(Q_{i}\right)\right\}}\left[D F_{j}\left(D_{k}\right) \times w t\left(D_{k}\right)\right]
$$

In general, any monotone function of the replacement probability could be used for $w t\left(D_{k}\right)$. For the experiments reported below, the weight is simply set to the best available estimate of the replacement probability.

Improbable translations that are common terms can also cause problems in equation (1), since common terms are likely to have higher $T F$ 's as well. One way to limit this effect is to use a weighted sum in the $T F$ computation:

$$
T F_{j}\left(Q_{i}\right)=\sum_{\left\{k \mid D_{k} \in T\left(Q_{i}\right)\right\}}\left[T F_{j}\left(D_{k}\right) \times w t\left(D_{k}\right)\right]
$$

Again, for the experiments reported below the replacement probability estimate is used as the weight. Finally, either $T F$ formula could be combined with any way of computing $D F$. In the experiments reported below, the following combinations were tried:

\begin{tabular}{|c|c|c|}
\hline Method & $\begin{array}{c}\boldsymbol{T F} \\
\text { Formula }\end{array}$ & $\begin{array}{c}\boldsymbol{D F} \\
\text { Formula }\end{array}$ \\
\hline Pirkola & $(1)$ & $(2)$ \\
\hline Kwok & $(1)$ & $(4)$ \\
\hline MDF & $(1)$ & $(5)$ \\
\hline WDF & $(1)$ & $(6)$ \\
\hline WTF & $(7)$ & $(4)$ \\
\hline WTF/DF & $(7)$ & $(6)$ \\
\hline
\end{tabular}

Another way of leveraging information about replacement probabilities is to simply ignore the least likely replacements. Such an approach potentially offers two potential insights. First, it can reveal the extent of the adverse effect of lowprobability replacements on each technique. Second, it offers a principled way of tuning the degree of comprehensiveness of the dictionary to optimize the retrieval effectiveness of each technique. Two teams (from the University of Massachusetts [9] and the University of Maryland [2]) tried variants of this approach for TREC 2002 CLIR track. For the experiments reported below, a greedy technique was used in which replacements were retained in order of decreasing probability until a preset threshold on the cumulative probability was first exceeded. This approach guarantees that at least one replacement is retained. Mean uninterpolated average precision is reported for every threshold value between 0.1 and 1.0 , in increments of 0.1 . The experiments were run using a modified version of (**removed for blind reviewing**), which is a vector space retrieval system that was developed locally using Okapi BM-25 weights. Reported statistical significance tests were performed using a paired two-tailed $t$-test and are reported as significant for values of $p<0.05$.

\section{CLIR}

The CLIR experiments reported in this section were performed using the TREC 2002 CLIR track collection, which contains 383,872 articles from the Agence France Press (AFP) Arabic newswire, 50 topic descriptions written in English, and associated relevance judgments [12]. Queries were formed automatically using all the words in the title field of the topic description, which is designed to be representative of the style of queries typically issued in Web search applications. The documents were stemmed using $\mathrm{Al}$-Stem (a standard resource for the TREC CLIR track), diacritics were removed, and normalization was performed to convert the letters ya (ي) and alef maqsoura (ى) to ya (ي) and all the variants of alef (I) and hamza (๕), namely alef ('), alef hamza (إ), alef maad (I), hamza ( $)$ ), waw hamza (g), and ya hamza (s), to alef (l). The English queries were stemmed before translation using the Porter stemmer for compatibility with the translation resources described below.

\subsection{Estimating Replacement Probabilities}

Five translation resources of three types were combined for the application. Combining resources is useful, because (a) the coverage of the combined resources is typically better than any of the individual resources, and (b) combining resources can serve to reinforce good translations. The resources were as follows:

1. Two bilingual term lists that were constructed using two Web-based machine translation systems (Tarjim and AlMisbar [16][17]). In each case, sets of isolated unique English words found in a $200 \mathrm{MB}$ collection of Los Angeles Times news stories [10] were submitted for translation from English into Arabic. Each system returned at most one translation for each submitted word. Together, the two term lists covered about $15 \%$ of the unique Arabic stems in the TREC collection (measured by using Al-Stem on both the term list and the collection). 
2. The Salmone Arabic-to-English dictionary (from Tufts University), from which we extracted only the translations. No translation preference information is indicated in this dictionary. The coverage of the resulting term list, measured in the same way, was about $7 \%$ of the unique Arabic stems in the TREC collection.

3. Two translation probability tables, one for English-toArabic and one for Arabic-to-English. These tables were constructed from tables provided by BBN, which were in turn constructed from a large collection of aligned English and Arabic United Nations documents using the Giza++ implementation of IBM's model 1 statistical machine translation design. The coverage of the Arabicto-English table, measured in the same way, was $29 \%$ of the unique Arabic stems in the TREC collection.

These translation resources were combined in the following manner:

1. All resources that were originally provided as Arabic-toEnglish were inverted. For the translation probability table, the probabilities for each translation pair were retained and then the inverted tables were renormalized so that the values of the "probabilities" for each sourcelanguage term summed to one. This process likely introduced some error, since probabilities for rare events may not have been accurately estimated.

2. A uniform distribution was used to assign probabilities to the translations obtained from machine translation systems and the Salmone dictionary. Tarjim and AlMisbar each returned at most one translation for an English word, but two English words might share a common translation. When $n$ alternatives were known from a single source, each was assigned a probability of $1 / n$.

3. The resulting translation probabilities were then combined by summing the probabilities for a given Arabic translation across the sources in which it appeared and then dividing by the number of sources in which the English term had appeared. For example, if Tarjim, AlMisbar and Salmone contained the English term, with Tarjim containing some specific translation with probability 1.0, Al-Misbar lacking that translation (i.e., assigning it a probability of 0.0 ), and Salmone assigning it a probability of 0.5 (because two translations were known), then the resulting combined probability would be $1 / 3+0 / 3+0.5 / 3=0.5$.

The resulting translation resource contained what appeared to be reasonable estimates of translation probabilities, and covered $36 \%$ of the unique Arabic stems in the TREC collection.

\subsection{Results}

Figure 1 shows the mean uninterpolated average precision for each of the six structured query methods for each threshold value and Table 1 shows the same results in tabular form. As a baseline, one-best query translation (using only the most likely translation) was also run. This widely reported baseline seems appropriate in this case because any cumulative probability threshold will result in use of at least the most probable translation for each query term. Kwok's and Pirkola's methods turned out to be essentially indistinguishable, with MDF method performing nearly as well (statistically significantly worse only at threshold values of 0.2 and 0.3 ).
The WTF/DF method produced results that were statistically significantly better than the one-best baseline for every threshold value except 0.1 and 1.0. Moreover, WTF/DF was the only one of the probabilistic techniques that did not exhibit a dramatic decrease in effectiveness as the threshold increased. The best WTF/DF result (at a threshold of 0.6) is statistically indistinguishable from the best result of Pirkola, Kwok, or WTF (in each case, at a threshold of 0.4), but the reduced dependence on accurate tuning of the threshold makes WTF/DF clearly the preferred method.

Table 1: CLIR: Mean average precision, title queries. Black (gray) cells represent statistically better (worse) results, compared to the one-best translation baseline.

Cumulative Probability Threshold - CLIR

\begin{tabular}{|l|l|l|l|l|l|l|l|l|l|}
\hline 0.1 & 0.2 & 0.3 & 0.4 & 0.5 & 0.6 & 0.7 & 0.8 & 0.9 & 1.0 \\
\hline
\end{tabular}

\begin{tabular}{|l|l|l|l|l|l|l|l|l|l|l|}
\hline Baseline & 0.16 & 0.16 & 0.16 & 0.16 & 0.16 & 0.16 & 0.16 & 0.16 & 0.16 & 0.16 \\
\hline
\end{tabular}

\begin{tabular}{l|l|l|l|l|l|l|l|l|l|l|}
\hline Pirkola & 0.16 & 0.16 & 0.17 & 0.19 & 0.17 & 0.14 & 0.09 & 0.08 & 0.07 & 0.01 \\
\hline
\end{tabular}

\begin{tabular}{|c|c|c|c|c|c|c|c|c|c|c|c|c|}
\hline Kwok & 0.16 & 0.16 & 0.17 & 0.18 & 0.17 & 0.14 & 0.10 & 0.08 & 0.07 & 0.00 \\
\hline
\end{tabular}

\begin{tabular}{l|l|l|l|l|l|l|l|l|l|l|} 
MDF & 0.16 & 0.16 & 0.17 & 0.18 & 0.16 & 0.13 & 0.08 & 0.08 & 0.07 & 0.02 \\
\hline
\end{tabular}

\begin{tabular}{l|l|l|l|l|l|l|l|l|l|l|} 
WTF & 0.16 & 0.17 & 0.18 & 0.19 & 0.18 & 0.16 & 0.12 & 0.11 & 0.11 & 0.03 \\
\hline WDF & 0.16 & 0.16 & 0.17 & 0.17 & 0.16 & 0.13 & 0.09 & 0.11 & 0.10 & 0.07 \\
\hline
\end{tabular}

\begin{tabular}{l|l|l|l|l|l|l|l|l|l|l|} 
WDF & 0.16 & 0.16 & 0.17 & 0.17 & 0.16 & 0.13 & 0.09 & 0.11 & 0.10 & 0.07 \\
\hline
\end{tabular}

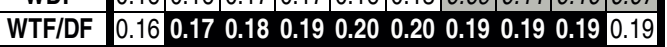

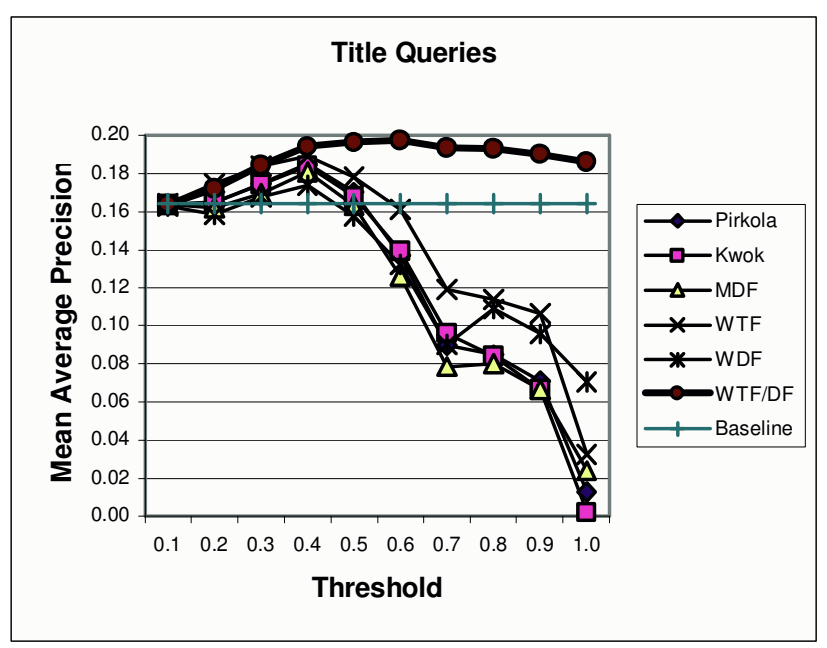

Figure 1: CLIR: Dependence of retrieval effectiveness on cumulative probability threshold, title queries.

\section{OCR-BASED RETRIEVAL}

Previous approaches to retrieval of OCR-degraded text have focused primarily on correcting OCR errors [7][15] or on fuzzy matching techniques that are less sensitive than exact string matching to OCR errors [1][5]. This section demonstrates the generality of the query-time replacement techniques developed above, using them to combine $T F$ and $D F$ evidence for a novel technique which attempts to replace each query term with possible OCR-distortions of the term and to estimate probability of the replacements.

The experiments were conducted with the Zad collection, which was obtained from the University of Maryland [3]. The collection is comprised of 2,730 documents extracted from Zad $A l-M e ' a d$, a printed book for which an accurately character coded electronic version (the "clean text") is also available [3]. Three sets of OCR outputs for the same documents were 
available: print resolution $(300 \times 300$ dots per inch (dpi)) as originally scanned, and down sampled versions at fine fax resolution (200x200 dpi) and standard fax resolution (200x100 dpi). The test collection includes 25 written topic descriptions and associated relevance judgments. Characters normalizations were performed as described above, and character 3-grams (3g) or character 4-grams (4g) were indexed. Darwish and Oard found those index terms to be among the most effective of OCR-based retrieval of Arabic [3].

\subsection{Estimating Replacement Probabilities}

Term replacement probabilities were estimated using a position-sensitive unigram character distortion model trained on 5,000 words of aligned clean and distorted texts from the collections being searched. The alignment was designed to simulate manual error correction of a small portion of the collection. $^{2}$ Since the appearance of Arabic characters varies by position, the standard four character positions (beginning, middle, end, isolated) were modeled.

Formally, given a clean word with characters $C_{l} . . C_{i} . . C_{n}$ and the resulting word after OCR degradation $D_{1} . . D_{j} . . D_{m}$, where $D_{j}$ resulted from $C_{i}, \varepsilon$ is the null character, $L$ is the position of the letter in the word (beginning, middle, end, or isolated), and \# is the word boundary, the three edit operations for the models would be:

$$
\begin{aligned}
& \mathrm{P}_{\text {substitution }}\left(C_{i} \rightarrow D_{j} \mid L\right)=\frac{\operatorname{count}\left(C_{i} \rightarrow D_{j} \mid L\right)}{\operatorname{count}\left(C_{i} \mid L\right)} \\
& \mathrm{P}_{\text {deletion }}\left(C_{i} \rightarrow \varepsilon \mid L\right)=\frac{\operatorname{count}\left(C_{i} \rightarrow \varepsilon \mid L\right)}{\operatorname{count}\left(C_{i} \mid L\right)} \\
& \mathrm{P}_{\text {insertion }}\left(\varepsilon \rightarrow D_{j} \mid L\right)=\frac{\operatorname{count}\left(\varepsilon \rightarrow D_{j} \mid L\right)}{\operatorname{count}\left(C_{i} \mid L\right)}
\end{aligned}
$$

If the count in the numerator was zero, the computation would be repeated without conditioning on position. If the count remained zero, a value of zero was recorded.

A separate model was trained for each resolution. Two factors made automatic alignment of the OCR output to the clean text challenging. First, the printed and clean text versions in the Zad collection were obtained from different sources that exhibited minor differences (mostly substitution or deletion of particles such as in, from, or, and then). Second, some areas in the scanned images of the printed page exhibited image distortions that resulted in relatively long runs of OCR errors. The alignment was performed using SCLITE from the National Institute of Standards and Technology (NIST). SCLITE employs a dynamic programming string alignment algorithm, which attempts to minimize the Levenshtein distance (edit distance) between two strings. Conceptually, the algorithm uses identical matches to anchor alignment, and then uses word position with respect to those anchors to estimate an optimal alignment on the remainder of the words.

SCLITE was originally developed for speech recognition applications, but in OCR applications additional characterlevel evidence is available. SCLITE alignments were therefore accepted only if the number of character edit operations were

\footnotetext{
${ }^{2}$ Smaller and larger training sets were tried, but no
} improvement resulted from more than 5,000 words. less than or equal to $50 \%$ of the length of the shorter of the two matched words. To align the words that were not aligned by SCLITE the following algorithm was used:

1. Using the existing alignments as anchors, given an unaligned word at position $l$ from the preceding anchor in a clean document, sequentially compare it to the words, in the corresponding degraded document between the corresponding pair of anchors with position $l$ ' from the preceding anchor where $\left|l^{\prime}-l\right| \leq 5$.

2. When comparing two words, if the difference between their respective word lengths was less than or equal to 2 characters and the number of edit operations between the two words (using Lenvenshtien's edit distance) was less than a certain percentage $q$ of the word length of the shorter one (the percentage $q$ was the number of edit operation divided by the length of the shorter word), then the newly aligned words were used as anchors. Initially, $q$ was set to $60 \%$.

3. Steps 1 and 2 were iterated two more times using the new anchors with $q$ equal to $40 \%$ and $20 \%$ to attempt to find more alignments.

This alignment technique works well for print resolution, but it is a significant source of errors for highly degraded cases (e.g., standard fax resolution).

Given a pair of aligned words, they were aligned at the character level by finding the edit distance between them using the Levenshtein edit distance algorithm and then back tracing the algorithm to identify insertions, deletions, and substitutions.

The resulting model was then used to assign a probability to possible distortions of each query term as follows:

1. For each character in a clean query term, generate all substitutions or deletions that have non-zero probability (i.e., were observed at least once in the training data). The unchanged character is generated at this step as a substitution.

2. For each possible insertion point, generate all possible single insertions. Possible insertion points are before the first character, between any pair of characters, and after the last character. A null insertion is generated at each point to cover the remainder of the probability mass.

3. For each string that could result from the power set of all possible substitutions or deletions and all possible insertions, compute the probability of generating that string as the product of the associated insertion, substitution, and deletion probabilities.

A more efficient implementation would be desirable in an operational setting, but this approach suffices for the experiments reported below.

\subsection{Results}

Figure 2 shows the mean uninterpolated average precision at print resolution for each of the six structured query methods for each threshold value and Table 2 shows the same data in tabular form. Figure 3 and Table 3 present the corresponding results for fine fax resolution. As a baseline, the same index terms $(3 \mathrm{~g}$ or $4 \mathrm{~g}$ ) were run with the clean (undistorted) queries, since any cumulative probability threshold results in a superset of that baseline case. 


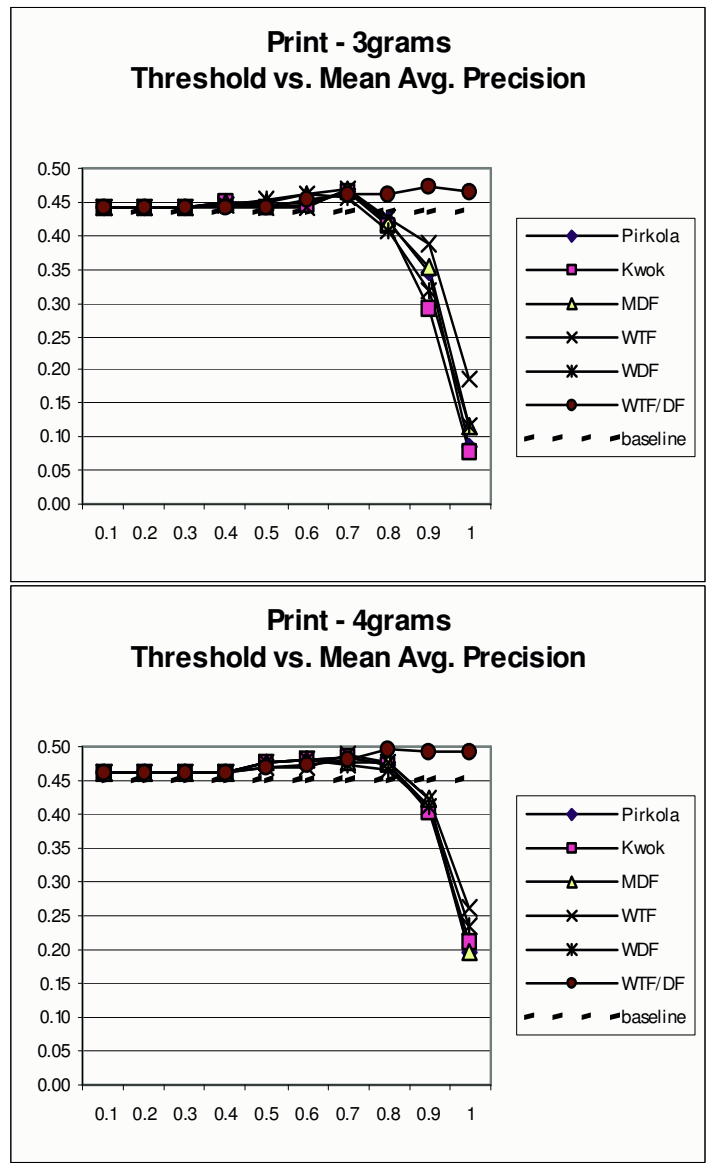

Figure 2: Print: Dependence of retrieval effectiveness on cumulative probability threshold, title queries.

No statistically significant differences were observed at any resolution or threshold value between the Pirkola, Kwok and MDF methods, which tends to confirm the observation made in the CLIR application that the simpler implementation of Kwok's method results in no significant adverse effect on retrieval effectiveness. For print resolution, every structured query technique achieved a statistically significant improvement over the baseline when used with the better of the two indexing terms (4g). Among these, WTF/DF both achieved the greatest improvement $(9.7 \%$ relative), and exhibited the greatest range of threshold values over which the improvement was statistically significant (0.6 to 1.0). Therefore, as with CLIR, WTF/DF is clearly the preferred technique in this application.

No statistically significant improvements over the baseline were observed for the fine fax resolution or the standard fax resolution (not shown). This may, however, reflect errors in the alignment of the training data rather than limitations in the replacement techniques that was tried. The same general trends are observable in Figure 3 as in Figure 2, so the use of WTF/DF is certainly not counterindicated for the fine fax condition.

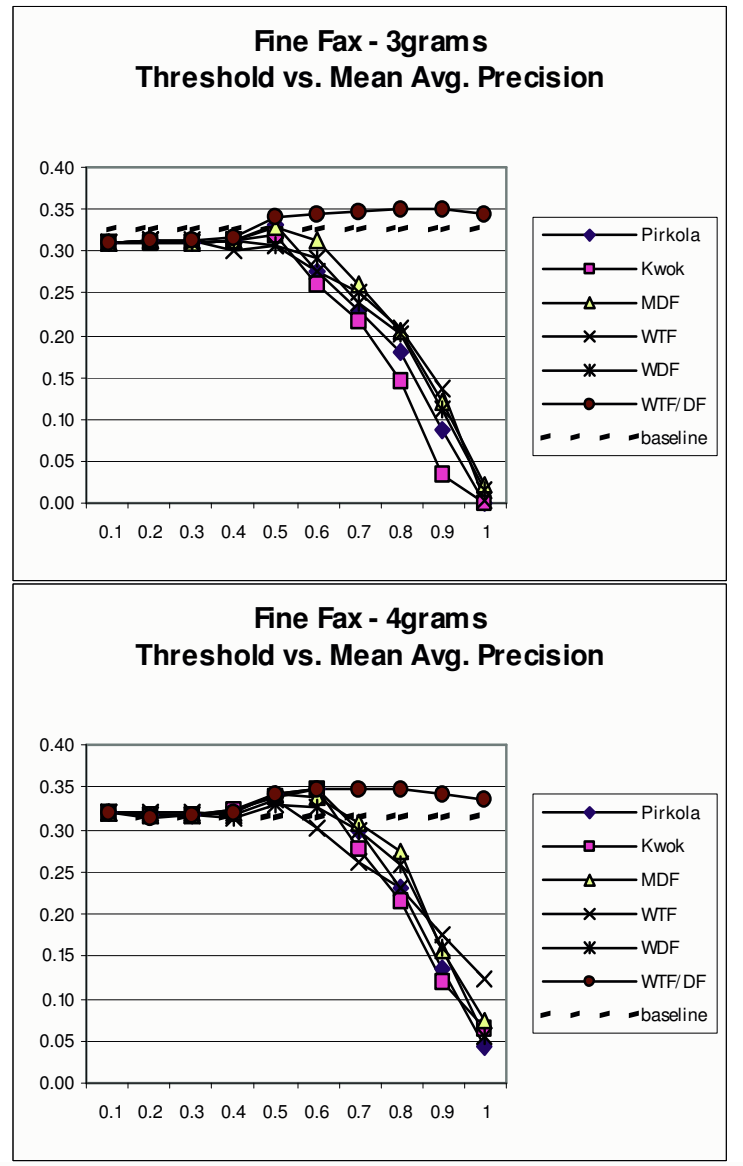

Figure 3: Fine fax: Dependence of retrieval effectiveness on cumulative probability threshold, title queries.

Table 2: Print: Mean average precision, title queries. Black (gray) cells represent statistically better (worse) results, compared to the clean query baseline.

\begin{tabular}{|l|l|l|l|l|l|l|l|l|l|}
\hline \multicolumn{7}{|c|}{ Cumulative Probability Threshold - Print } \\
\hline 0.1 & 0.2 & 0.3 & 0.4 & 0.5 & 0.6 & 0.7 & 0.8 & 0.9 & 1.0 \\
\hline
\end{tabular}

\begin{tabular}{ll|l|l|l|l|l|l|l|l|l|l|}
\hline Baseline & 0.43 & 0.43 & 0.43 & 0.43 & 0.43 & 0.43 & 0.43 & 0.43 & 0.43 & 0.43 \\
\hline
\end{tabular} \begin{tabular}{ll|l|l|l|l|l|l|l|l|l|l|l|}
\hline Pirkola & 0.44 & 0.44 & 0.44 & 0.45 & 0.44 & 0.45 & 0.47 & 0.42 & 0.35 & 0.09 \\
\hline
\end{tabular} \begin{tabular}{l|l|l|l|l|l|l|l|l|l|l|l|l|l|l|l}
\hline Kwok & 0.44 & 0.44 & 0.44 & 0.45 & 0.44 & 0.44 & 0.47 & 0.42 & 0.29 & 0.08 \\
\hline
\end{tabular}

\begin{tabular}{ll|l|l|l|l|l|l|l|l|l|l|l|l}
\hline $3 g$ & MDF & 0.44 & 0.44 & 0.44 & 0.45 & 0.45 & 0.46 & 0.47 & 0.42 & 0.35 & 0.12 \\
\hline
\end{tabular}

\begin{tabular}{l|l|l|l|l|l|l|l|l|l|l|l|l|l|l}
\hline WTF & 0.44 & 0.44 & 0.44 & 0.44 & 0.44 & 0.44 & 0.47 & 0.43 & 0.39 & 0.18 \\
\hline
\end{tabular}

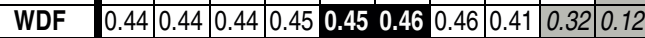
\begin{tabular}{l|l|l|l|l|l|l|lllllll} 
WTF/DF & 0.44 & 0.44 & 0.44 & 0.44 & 0.44 & 0.45 & 0.46 & 0.46 & 0.47 & 0.47
\end{tabular}

\begin{tabular}{|l|l|l|l|l|l|l|l|l|l|l|}
\hline Baseline & 0.45 & 0.45 & 0.45 & 0.45 & 0.45 & 0.45 & 0.45 & 0.45 & 0.45 & 0.45 \\
\hline
\end{tabular}

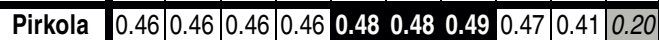

\begin{tabular}{l|l|l|l|l|l|lllllll} 
Kwok & 0.46 & 0.46 & 0.46 & 0.46 & $\mathbf{0 . 4 8}$ & $\mathbf{0 . 4 8}$ & $\mathbf{0 . 4 9}$ & 0.47 & 0.40 & 0.21 \\
\hline
\end{tabular}

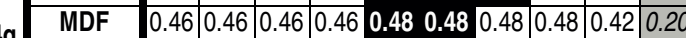

\begin{tabular}{l|l|l|l|l|l|l|l|l|l|l|l|l|l|l|l|l|l} 
WTF & 0.46 & 0.46 & 0.46 & 0.46 & 0.47 & 0.47 & 0.49 & 0.48 & 0.42 & 0.26 \\
\hline
\end{tabular}

\begin{tabular}{l|l|l|l|l|l|l|l|l|l|l|l} 
WDF & 0.46 & 0.46 & 0.46 & 0.46 & $\mathbf{0 . 4 8}$ & 0.48 & 0.47 & 0.47 & 0.41 & 0.23 \\
\hline
\end{tabular}

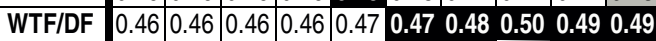


Table 3: Fine fax: Mean average precision, title queries. Black (gray) cells represent statistically better (worse) results, compared to the clean query baseline.

\begin{tabular}{|c|c|c|c|c|c|c|c|c|c|c|c|}
\hline & \multicolumn{10}{|c|}{ Cumulative Probability Threshold - Fine Fax } \\
\hline & & 0.1 & 0.2 & 0.3 & 0.4 & 0.5 & 0.6 & 0.7 & 0.8 & 0.9 & 1.0 \\
\hline & Baseline & 0.32 & 0.32 & 0.32 & 0.32 & 0.32 & 0.32 & 0.32 & 0.32 & 0.32 & 0.32 \\
\hline & Pirkola & 0.31 & 0.31 & 0.31 & 0.31 & 0.33 & 0.27 & 0.23 & 0.18 & 0.09 & 0.00 \\
\hline & Kwok & 0.31 & 0.31 & 0.31 & 0.31 & 0.32 & 0.26 & 0.22 & 0.14 & 0.04 & 0.00 \\
\hline & MDF & 0.31 & 0.31 & 0.31 & 0.31 & 0.33 & 0.31 & 0.26 & 0.20 & 0.12 & 0.02 \\
\hline & WTF & 0.31 & 0.31 & 0.31 & 0.30 & 0.31 & 0.28 & 0.25 & 0.21 & 0.13 & 0.00 \\
\hline & WDF & 0.31 & 0.31 & 0.31 & 0.31 & 0.31 & 0.29 & $\mid$\begin{tabular}{|l|} 
\\
\end{tabular} & 0.20 & 0.11 & 0.02 \\
\hline & WTF/DF & 0.31 & 0.31 & 0.31 & 0.32 & 0.34 & 0.34 & 0.35 & 0.35 & 0.35 & 0.34 \\
\hline & Baseline & 0.31 & 0.31 & 0.31 & 0.31 & 0.31 & 0.31 & 0.31 & 0.31 & 0.31 & 0.31 \\
\hline \multirow{6}{*}{4} & Pirkola & 0.32 & 0.32 & 0.32 & 0.32 & 0.34 & 0.35 & 0.30 & 0.23 & 0.14 & 0.04 \\
\hline & Kwok & 0.32 & 0.32 & 0.32 & 0.32 & 0.34 & 0.35 & 0.28 & 0.21 & 0.12 & 0.07 \\
\hline & MDF & 0.32 & 0.32 & 0.32 & 0.32 & 0.34 & 0.34 & 0.31 & 0.27 & 0.16 & 0.07 \\
\hline & WTF & 0.32 & 0.32 & 0.32 & 0.32 & 0.33 & 0.30 & 0.26 & 0.23 & 0.17 & 0.12 \\
\hline & WDF & 0.32 & \begin{tabular}{|l|}
0.32 \\
\end{tabular} & \begin{tabular}{|l|}
0.32 \\
\end{tabular} & \begin{tabular}{|l|}
0.31 \\
\end{tabular} & 0.33 & 0.33 & 0.30 & 0.26 & 0.16 & 0.06 \\
\hline & WTF/DF & 0.32 & \begin{tabular}{|l|}
0.31 \\
\end{tabular} & \begin{tabular}{|l|}
0.32 \\
\end{tabular} & \begin{tabular}{|l|}
0.32 \\
\end{tabular} & 0.34 & 0.35 & 0.35 & 0.35 & 0.34 & \begin{tabular}{|l|}
0.34 \\
\end{tabular} \\
\hline
\end{tabular}

\section{CONCLUSION AND FUTURE WORK}

This paper has introduced a family of methods for query term replacement that exploit estimates of replacement probabilities while also incorporating the vector space model's concept of "document frequency." Both Kwok's method and MDF were found to achieve retrieval effectiveness values similar to that obtained with Pirkola's structured query method, so Kwok's method seems to be a good basis from which to build probabilistic structured query methods. Coverage of rare translations was shown to be problematic for all three methods, however. Use of only the most likely translations was found to be an effective and expedient, but only if an appropriate threshold on cumulative probability is used. Of the three probabilistic structured query methods introduced in this paper, WTF/DF was the clear winner, showing both the best retrieval effectiveness and the least sensitivity to the cumulative probability threshold. Finally, the novel approach of producing possible replacements for query terms that could have been generated by OCR proved to be a useful technique for improving retrieval of OCR-degraded text.

There are a number of interesting directions for future work suggested by these results:

1. Improved weighting techniques. The use of raw probability estimates as weights in the WTF/DF method seems intuitively appealing, but it is possible that using some function of the probabilities (e.g., $\log$ p) may actually outperform raw probabilities. There are also opportunities to explore better smoothing methods when estimating the probabilities.

2. Other applications. The WTF/DF method can be used in any application where replacement probabilities can be reliably estimated. Examples of potential application areas are thesaurus expansion, speech-based retrieval, statistical approximations of morphology, and perhaps gene sequence matching.

3. Structured document indexing. Query processing and document processing exhibit a strong duality, so it may be possible to leverage some of the techniques developed here at indexing time rather than query time for applications such as stemming, translation based indexing [11], speech retrieval and OCR-based retrieval.

Variants of query term replacement are important in several information retrieval applications, and access to reliable estimates of replacement probabilities from corpus statistics is becoming increasingly common. The techniques described in this paper balance effectiveness and efficiency in ways that are likely to prove immediately useful, and they should additionally serve as a solid basis for future research on this important problem.

\section{ACKNOWLEDGMENTS}

***Removed for blind reviewing***

\section{REFERENCES}

[1] Baeza-Yates, R. and G. Navarro, "A Faster Algorithm for Approximate String Matching." Proceedings of Combinatorial Pattern Matching (CPM'96), Springer-Verlag LNCS, v. 1075, pages 113, 1996.

[2] Darwish, K. and D. Oard, "CLIR Experiments at Maryland for TREC 2002: Evidence Combination for Arabic-English Retrieval," TREC 2002.

[3] Darwish, K. and D. Oard, "Term Selection for Searching Printed Arabic," SIGIR 2002, 261-268, 2002.

[4] Gey, F. and D. Oard, "The TREC-2001 CrossLanguage Information Retrieval Track: Searching Arabic Using English, French or Arabic Queries," TREC 2001, 16-25.

[5] Harding, S., W. Croft, and C. Weir, "Probabilistic Retrieval of OCR Degraded Text Using N-Grams." European Conference on Digital Libraries, 1997

[6] Hiemstra, D. Using language models for information retrieval Ph.D. Thesis University of Twente, Enschede, 2001.

[7] Hong, T., "Degraded Text Recognition Using Visual and Linguistic Context." Ph.D. thesis, Computer Science Department, SUNY Buffalo, 1995.

[8] Kwok, K. L., Personal communication.

[9] Larkey, L., J. Allen, M. E. Connell, A. Bolivar, and C. Wade, "UMass at TREC 2002: Cross Language and Novelty Tracks," TREC 2002.

[10] NIST, Text Research Collection Volume 5, April 1997.

[11] Oard, D. W. and F. Ertunc "Translation-Based Indexing for Cross-Language Retrieval," ECIR 2002: 324-333, 2002.

[12] Oard, D. W. and F. Gey, "The TREC-2002 Arabic/English CLIR Track," TREC 2002.

[13] Pirkola, A. "The Effects of Query Structure and Dictionary setups in DictionaryBased Cross-language Information Retrieval," Proceedings of the $21^{\text {st }}$ International ACM SIGIR Conference on Research and Development in Information Retrieval, pages 5563, 1998.

[14] Robertson, S. E., S. Walker, M. Hancock-Beaulieu, A. Gull, and M. Lau, "Okapi at TREC-3," In the Fourth Text REtrieval Conference (TREC-3), 73--96, 1996. 
[15] Taghva, K., J. Borsack, and A. Condit, "An Expert System for Automatically Correcting OCR Output." Proceedings of the SPIE - Document Recognition, pages 270--278, 1994.

[16] tarjim.ajeeb.com, Sakhr Technologies, Cairo, Egypt www.sakhr.com

[17] www.almisbar.com, ATA Software Technology Limited, North Brentford Middlesex, UK.

[18] Xu, J., Weischedel, R., and Nguyen, C. Evaluating a Probabilistic Model for Cross-lingual Information Retrieval. In Proceedings of SIGIR, 2001, pages 105-110, 2001. 